\title{
c-FLIP and CD95 signaling are essential for survival of renal cell carcinoma
}

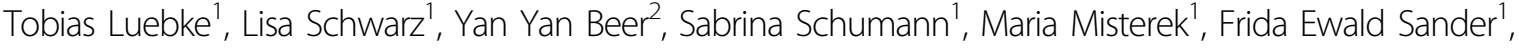 \\ Carlos Plaza-Sirvent ${ }^{1}$ and Ingo Schmitz ${ }^{1,2}$
}

\begin{abstract}
Clear cell renal cell carcinoma (ccRCC) is the most-prominent tumor type of kidney cancers. Resistance of renal cell carcinoma (RCC) against tumor therapy is often owing to apoptosis resistance, e.g., by overexpression of anti-apoptotic proteins. However, little is known about the role of the apoptosis inhibitor c-FLIP and its potential impact on death receptor-induced apoptosis in ccRCC cells. In this study, we demonstrate that c-FLIP is crucial for resistance against CD95L-induced apoptosis in four cCRCC cell lines. Strikingly, downregulation of c-FLIP expression by short hairpin RNA (shRNA)interference led to spontaneous caspase activation and apoptotic cell death. Of note, knockdown of all c-FLIP splice variants was required to induce apoptosis. Stimulation of $\mathrm{CCRCC}$ cells with CD95L induced NF-KB and MAP kinase survival pathways as revealed by phosphorylation of ReIA/p65 and Erk1/2. Interestingly, CD95L surface expression was high in all cell lines analyzed, and CD95 but not TNF-R1 clustered at cell contact sites. Downstream of CD95, inhibition of the NF-KB pathway led to spontaneous cell death. Surprisingly, knockdown experiments revealed that C-FLIP inhibits NF-KB activation in the context of CD95 signaling. Thus, C-FLIP inhibits apoptosis and dampens NFKB downstream of CD95 but allows NF-KB activation to a level sufficient for cCRCC cell survival. In summary, we demonstrate a complex CD95-FLIP-NF-KB-signaling circuit, in which CD95-CD95L interactions mediate a paracrine survival signal in ccRCC cells with c-FLIP and NF-KB both being required for inhibiting cell death and ensuring survival. Our findings might lead to novel therapeutic approaches of RCC by circumventing apoptosis resistance.
\end{abstract}

\section{Introduction}

Renal cell carcinoma (RCC) accounts for $3.8 \%$ of malignancies of the adult and over $90 \%$ of kidney tumors ${ }^{1}$. RCC is a cancer of the proximal renal tubular epithelium and the collecting tubular epithelium and comprises a variety of malignancies that differ in histology and on the molecular level ${ }^{1-3}$. One particular type of RCC, the clear cell RCC (ccRCC), often exhibits mutations in the von Hippel-Lindau (VHL) tumor suppressor gene and in PBRM1, a component of the PBAF chromatin-remodeling complex ${ }^{4}$. Another main characteristic of RCC is the

Correspondence: Ingo Schmitz (ingo.schmitz@helmholtz-hzi.de)

${ }^{1}$ Institute of Molecular and Clinical Immunology, Otto-von-Guericke University, Leipziger Straße 44, 39120 Magdeburg, Germany

${ }^{2}$ Systems-Oriented Immunology and Inflammation Research Group, Helmholtz Centre for Infection Research, Inhoffenstraße 7, 38124 Braunschweig, Germany Edited by T. Kaufmann resistance toward apoptosis-inducing chemotherapeutic approaches ${ }^{5}$. Therefore, previous therapies were based on cytokines such as interleukin- 2 or interferon- $\alpha$ to boost immune responses ${ }^{1,6}$. More recently, therapy switched to molecular approaches targeting multiple tyrosine kinases or the mammalian target of rapamycin ${ }^{1,6}$. Although the median survival time was increased by the molecular therapies, kidney cancer still accounted for 143,000 deaths in $2012^{2,6}$. Therefore, additional molecular therapies are urgently needed for the treatment of RCC.

Resistance to apoptosis is a hallmark of cancer cells and can lead to tumor formation ${ }^{7}$. One important molecular pathway, called extrinsic apoptosis, is initiated by death ligands like CD95L-, TNFo-, and TRAIL-triggering activation of their respective receptors ${ }^{8}$. Upon ligand binding, the protein Fas-associated protein with death domain (FADD) binds to CD95 and acts as recruitment platform

\section{(c) The Author(s) 2019}

(c) (i) Open Access This article is licensed under a Creative Commons Attribution 4.0 International License, which permits use, sharing, adaptation, distribution and reproduction cc) in any medium or format, as long as you give appropriate credit to the original author(s) and the source, provide a link to the Creative Commons license, and indicate if changes were made. The images or other third party material in this article are included in the article's Creative Commons license, unless indicated otherwise in a credit line to the material. If material is not included in the article's Creative Commons license and your intended use is not permitted by statutory regulation or exceeds the permitted use, you will need to obtain permission directly from the copyright holder. To view a copy of this license, visit http://creativecommons.org/licenses/by/4.0/. 
for death effector domain (DED)-containing proteins such as caspase- 8 (formerly known as FLICE) and caspase- 10 forming the death inducing signaling complex (DISC) ${ }^{8,9}$. When two caspase molecules come into close proximity, they undergo self-cleavage into an active form by removing the $\mathrm{N}$-terminal DED domains ${ }^{10,11}$. The activated caspase is then released from the DISC activating downstream effector caspases, leading to apoptotic death of the cell ${ }^{12,13}$.

The apoptotic machinery is controlled by various antiapoptotic proteins, whose expression is tightly regulated. For instance, cellular FLICE-like inhibitory proteins (cFLIP) can inhibit apoptosis at the DISC ${ }^{14}$. Three different c-FLIP splice variants (c-FLIP ${ }_{L}, c-$ FLIP $_{S}$, and c-FLIP ${ }_{\mathrm{R}}$ ) have been identified so far that are expressed on the protein level $^{14-17}$. All three c-FLIP proteins have, like caspase- 8 and caspase-10, two DEDs, which are crucial for DISC recruitment, inhibition of caspase activation and apoptosis execution ${ }^{14,18}$. Although c-FLIP is regarded primarily as an anti-apoptotic protein, the three isoforms can have different functions. Although only anti-apoptotic functions have been described for c-FLIP ${ }_{S}$ and c-FLIP $P_{R}$, cFLIP $_{L}$ was also shown to have pro-apoptotic features, which depend on the presence of the C-terminal caspaselike-domain that itself is catalytically inactive but allows activation of caspase- 8 when forming a heterodimer ${ }^{19,20}$. The different properties of $\mathrm{c}-\mathrm{FLIP}_{\mathrm{L}}$ in apoptosis regulation are determined by the expression levels of all c-FLIP splice variants as well as the stimulation strength of the death receptors ${ }^{21}$. In addition, when c-FLIP ${ }_{\mathrm{L}}$ is cleaved, a p43-FLIP fragment is generated, which was shown to interact with RIP1 and TRAF2 and thereby activating the nuclear factor " $\mathrm{K}$-light-chain-enhancer" of activated B cells $(\mathrm{NF}-\kappa \mathrm{B})^{22-24}$. On the other hand, c-FLIP has also been reported to inhibit death receptor-mediated NF- $\mathrm{KB}$ activation $^{25-27}$.

Anti-apoptotic proteins are known to be upregulated in several tumor types. For instance, inhibition of the extrinsic pathway allows cells having the potential for tumor formation to evade immune surveillance mechanisms, which recognize mutated and potentially defective cells $^{7}$. In this regard, upregulation of c-FLIP was for example shown in breast cancer ${ }^{28}$, melanoma ${ }^{17,29}$, and Hodgkin's lymphoma ${ }^{30,31}$. Furthermore, expression of cFLIP can be a resistance factor in colon cancer ${ }^{32}$, nonsmall cell lung cancer ${ }^{33}$, and urothelial carcinoma ${ }^{34}$. However, a contribution of c-FLIP splice variants in promoting apoptosis resistance in RCC has not been addressed in previous studies.

To uncover apoptosis resistance mechanisms in RCC, we characterized four different clear cell RCC (termed clearCa) cell lines, which are protected against TRAILmediated apoptosis ${ }^{35}$. Surprisingly, we found that clearCa cell lines critically depended on c-FLIP expression. In this regard, our study revealed a crucial function of c-FLIP and the CD95 system for survival of clearCa cells. Targeting this signaling axis may provide novel therapy options for ccRCC.

\section{Results \\ c-FLIP mediates resistance against CD95L-induced apoptosis}

We characterized the expression of DISC-related proteins in four different ccRCC cell lines (clearCa-2, -3, -4, and -6). Analysis of death receptor expression by surface staining revealed high levels of CD95 and intermediate TNF-R1 expression in all cell lines. TRAIL-R1 and TRAIL-R2 expression was detectable in clearCa-2, -3 , and -6 , whereas it was absent in clearCa-4 (Fig. 1a). The intracellular DISC components FADD and caspase- 8 were expressed equally between the four clearCa cell lines (Fig. 1b). The apoptosis inhibitor c-FLIP was expressed by all four cell lines analyzed, with c-FLIP ${ }_{\mathrm{L}}$ expression being higher than expression of the short splice variants c-FLIP and c-FLIP $P_{R}$ in clearCa-2 and -3 cell lines (Fig. 1b). Expression of the long and the short c-FLIP splice variants were comparable in clearCa-4 and clearCa-6. Genomic analysis of the 3' splicing site of intron 6 in the CFLAR gene revealed heterogeneous expression of the short splice variants $\mathrm{c}-\mathrm{FLIP}_{\mathrm{S}}$ and $\mathrm{c}-\mathrm{FLIP}_{\mathrm{R}}$. clearCa-3 and -6 were heterozygous for c-FLIP ${ }_{S}$ and c-FLIP ${ }_{R}$, clearCa-2 was homozygous for c-FLIP $P_{R}$ and clearCa-4 was homozygous

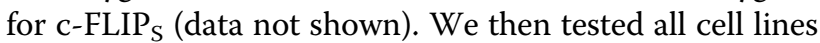
for CD95L-induced apoptosis. Cells were stimulated with 2 , 4 , or $10 \mathrm{ng} / \mathrm{mL}$ recombinant CD95L for $16 \mathrm{~h}$ in the presence or absence of the protein translation inhibitor cycloheximide (CHX) and cell death rates were measured by analyzing the sub-G1 DNA peak of propidium iodide stained cells. At the concentrations tested, all four cell lines were resistant against stimulation with CD95L alone, but were significantly sensitized by addition of CHX (Fig. 1c). Treatment of cells with CD95L alone was not sufficient for caspase-8 activation. Upon treatment of clearCa cells with $\mathrm{CHX}$, we detected distinct downregulation of the short-lived c-FLIP proteins (Fig. 2a). In contrast, expression of XIAP was only marginally affected and Bcl$\mathrm{x}_{\mathrm{L}}$ was downregulated in only some of the cell lines analyzed (Fig. 2a). As c-FLIP blocks CD95L-induced apoptosis at the level of the DISC, it is the most likely candidate for promoting CD95L-induced apoptosis resistance in clearCa cell lines. In line, combined stimulation of clearCa cells with CD95L and CHX revealed loss of c-FLIP expression, activation of caspase- 8 and PARP cleavage (Fig. 2b). Moreover, downregulation of c-FLIP proteins upon $\mathrm{CHX}$ treatment preceded activation of caspase- 8 and caspase-3 (Fig. 2c). 

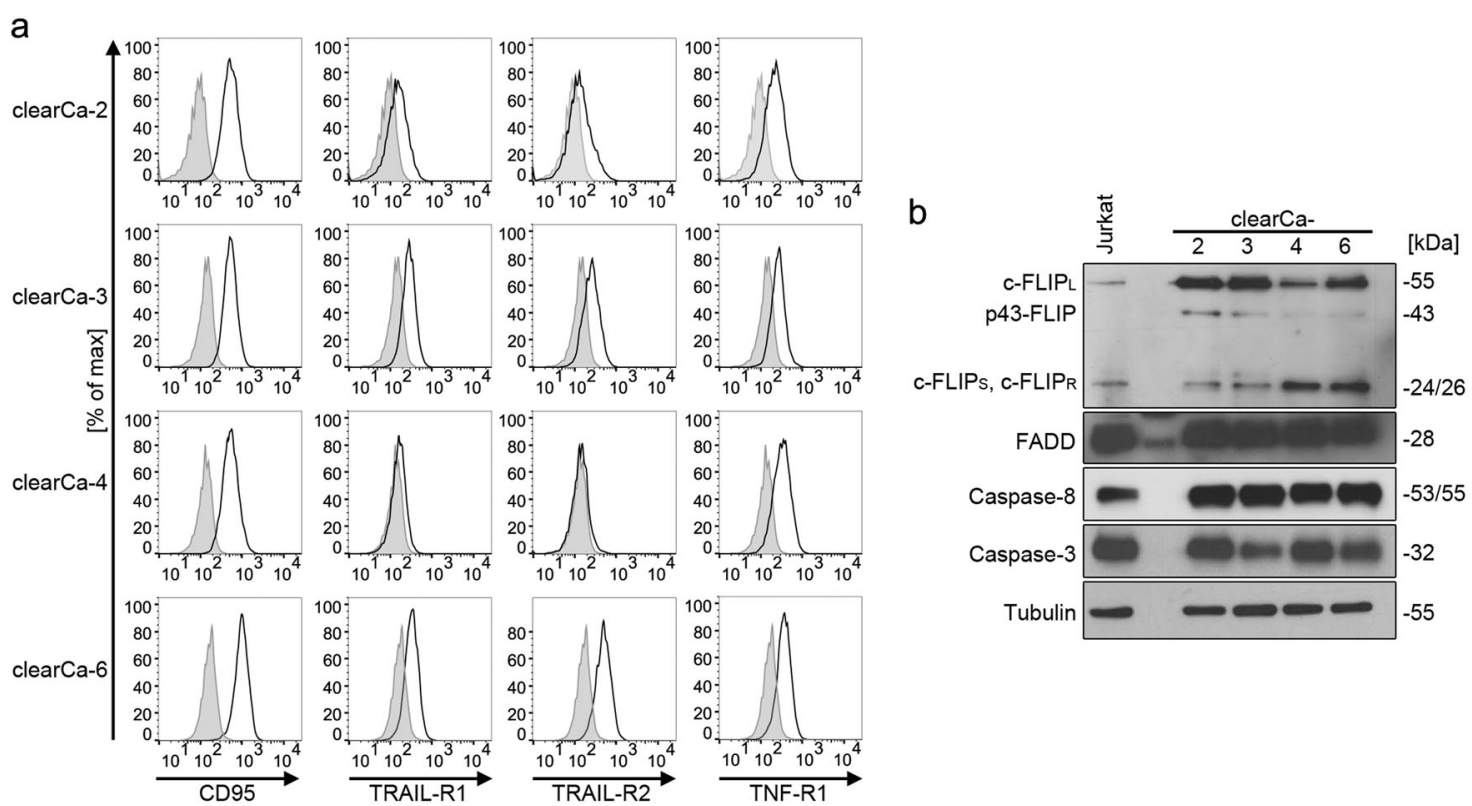

C

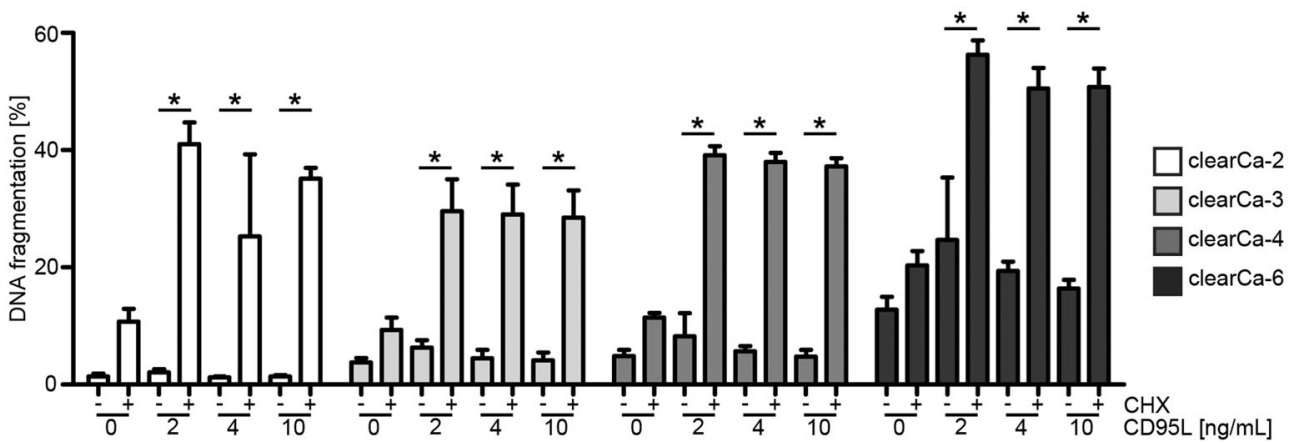

Fig. 1 Cycloheximide sensitizes clearCa cells towards CD95L-induced apoptosis. a Surface expression of death receptors CD95, TRAIL-R1, TRAILR2, or TNF-R1 (black line) on clearCa-2, $-3,-4$, and -6 cells was detected by flow cytometry with specific antibodies. Unstained samples are shown in gray. $\mathbf{b}$ Expression levels of the DISC proteins C-FLIP, FADD, and caspase-8 as well as caspase-3 in clearCa-2, $-3,-4$, and -6 cells were analyzed via immunoblotting. Tubulin served as loading control. c Analysis of DNA fragmentation after stimulation of clearCa cells with $0,2,4$, or $10 \mathrm{ng} / \mathrm{mL}$ CD95L in the presence or absence of $10 \mu \mathrm{g} / \mathrm{mL} \mathrm{CHX}$ for $16 \mathrm{~h}$. Bars display the mean of at least three experiments, error bars represent SD. Statistical significances were calculated by one-tailed Mann-Whitney $U$ test; * $p \leq 0.05$

\section{c-FLIP is essential for survival of clearCa cell lines}

To analyze the impact of c-FLIP on mediating apoptosis resistance, clearCa-4 was chosen for further analysis. clearCa-4 cells expressed comparable amounts of c-FLIP ${ }_{\mathrm{L}}$ and c-FLIP and were the only cell line in the panel that lacked c-FLIP $P_{R}$ expression against which no short hairpin RNA (shRNA) exists. We employed a shRNA approach for the downregulation of particular c-FLIP splice variants to understand potential functional differences between cFLIP $_{\mathrm{L}}$ and c-FLIP in apoptosis resistance. clearCa-4 cells were treated with lentiviral constructs, targeting either $\mathrm{c}$ $\operatorname{FLIP}_{\mathrm{L}}\left(\Delta \mathrm{c}-\mathrm{FLIP}_{\mathrm{L}}\right)$, c-FLIP $\left(\Delta \mathrm{c}-\mathrm{FLIP}_{\mathrm{S}}\right)$, or all splice variants c-FLIP ${ }_{L}$, c-FLIP , and c-FLIP $\left(\Delta c-\right.$ FLIP $\left._{L / S}\right)$ or containing a scrambled shRNA. All three c-FLIP-targeting constructs were potent in downregulating their respective targeted isoform, as can be seen by the diminished expression of c-FLIP ${ }_{\mathrm{L}}$, c-FLIP , and c-FLIP plus c-FLIP $_{\mathrm{S}}$, respectively (Fig. 3a). Downregulation of c-FLIP ${ }_{L}$ or cFLIP $_{S}$ alone showed no effect on caspase activation and survival of clearCa- 4 cells even at day 5 post transduction (data not shown). Surprisingly, $\Delta \mathrm{c}-\mathrm{FLIP}_{\mathrm{L} / \mathrm{S}}$ cells showed spontaneous caspase- 8 and caspase- 3 activation at day 3 post shRNA delivery (Fig. 3a). No differences in $\Delta c$-FLIP ${ }_{L}$ or $\Delta \mathrm{c}$-FLIP ${ }_{\mathrm{S}}$ cells were detectable, suggesting that apoptosis resistance is equally mediated by both splice variants. Microscopic analysis revealed high rates of dead cells with apoptotic morphological features such as membrane blebbing for $\Delta \mathrm{c}$-FLIP $\mathrm{L}_{\mathrm{S}}$ treated cells (Fig. 3b). In addition, we analyzed DNA fragmentation after 3 days of treatment, which was significantly elevated in $\Delta \mathrm{c}-\mathrm{FLIP}_{\mathrm{L} / \mathrm{S}}$ cells but not single knockdown cells (Fig. 3c), supporting the notion that the presence of one c-FLIP splice variant is 


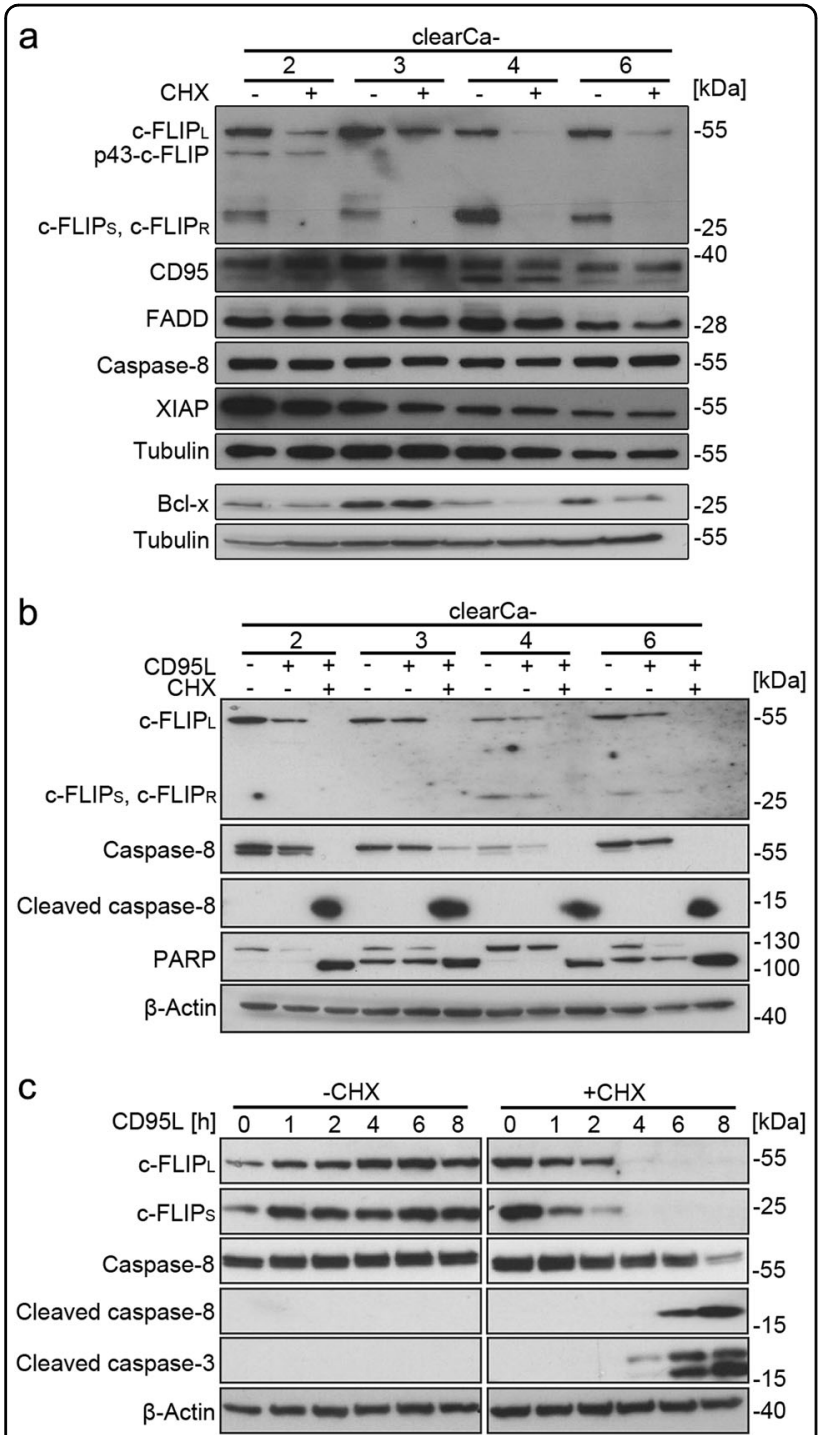

Fig. 2 Identification of c-FLIP as the cycloheximide-sensitive resistance factor in clearCa cells. a Analysis of the influence of cycloheximide on the expression levels of anti-apoptotic proteins. Cells were treated with $10 \mu \mathrm{g} / \mathrm{mL} \mathrm{CHX}$ for $8 \mathrm{~h}$ and analyzed by immunoblotting for expression of the indicated proteins. Tubulin was used as loading control. b Immunoblot analysis of c-FLIP expression, caspase-8 activation and PARP cleavage upon CD95L-induced apoptosis after stimulation with $10 \mathrm{ng} / \mathrm{mL}$ CD95L in the presence or absence of $10 \mu \mathrm{g} / \mathrm{mL} \mathrm{CHX}$ for $8 \mathrm{~h}$. $\beta$-Actin was used as loading control. c clearCa-4 cells were treated with $10 \mathrm{ng} / \mathrm{mL}$ CD95L in the presence or absence of $10 \mu \mathrm{g} / \mathrm{mL}$ CHX for up to $8 \mathrm{~h}$. Activation of caspase- 8 and caspase-3 as well as c-FLIP expression was analyzed by immunoblot analysis. $\beta$-Actin was used as loading control

sufficient to prevent spontaneous cell death. To proof an apoptotic mode of action, $\Delta \mathrm{c}$-FLIP $\mathrm{L}_{\mathrm{L} / \mathrm{S}}$ cells were additionally treated with the caspase inhibitor QVD-OPh (QVD) or the necroptosis inhibitor Necrostatin-1 (Nec-1) and analyzed 3 days later by flow cytometry. Treatment of cells with QVD completely blocked intracellular caspase-3 activation, whereas Nec-1 had no effect on caspase-3 activation (Fig. 3d). We also studied loss of plasma membrane asymmetry by staining of phosphatidylserine with fluorescent AnnexinV, followed by flow cytometric analysis, in wild type, scrambled shRNA-treated, $\Delta \mathrm{c}$ FLIP $_{\mathrm{L}}, \Delta \mathrm{c}-\mathrm{FLIP}_{\mathrm{S}}$, and $\Delta \mathrm{c}-\mathrm{FLIP}_{\mathrm{L} / \mathrm{S}}$ cells. Elevated numbers of AnnexinV-positive cells were detectable in $\Delta \mathrm{c}-\mathrm{FLIP}_{\mathrm{L} / \mathrm{S}}$ compared with wild-type cells (Fig. 3e). To follow the course of apoptosis, complete disintegration of the cell membrane was shown by concurrent DNA staining with 7AAD. As for AnnexinV, 7AAD-positive cells were detectable in $\Delta \mathrm{c}$-FLIP $\mathrm{L} / \mathrm{S}$ cells only (Fig. 3e). Complete loss of c-FLIP also induced spontaneous apoptosis of clearCa2 and clearCa-3 cells, showing a dependency on c-FLIP of these cell lines as well (Fig. 3f). Taken together, loss of cFLIP leads to spontaneous apoptosis in clearCa cell lines, whereas expression of one isoform is sufficient for cell viability. Thus, splice variant-independent c-FLIP expression seems to be necessary for clearCa survival in three of four cell lines (clearCa-2, -3, and -4).

\section{Re-expression of c-FLIP $P_{L}$ restores cell viability of $\Delta c-F L I P_{L / s}$ cells to wild-type levels}

To exclude any effects of the lentiviral treatment on cell survival or side effects of the used shRNA on other essential survival factors, we generated a construct to reexpress a shRNA-resistant form of c-FLIP $\mathrm{L}_{\mathrm{L}}$ while blocking the expression of wild-type c-FLIP ${ }_{\mathrm{L}}$ and c-FLIP $\mathrm{S}_{\mathrm{S}}$. For reexpression, we inserted silent mutations in the shRNA target sequence of the $\mathrm{c}$-FLIP $\mathrm{L}_{\mathrm{L}}$ gene and exchanged the resistance cassette against the mutated $c-F_{L I P}$ cDNA $\left(\Delta \mathrm{c}-\mathrm{FLIP}_{\mathrm{L} / \mathrm{S}}+\mathrm{c}\right.$-FLIP $\left.\mathrm{RESIST}\right)$. The capability of the construct to downregulate wild-type c-FLIP mRNA and simultaneous re-expression of c-FLIP RESIST $_{\text {mRNA was }}$ confirmed via quantitative real-time PCR with primers specific for endogenous and shRNA-resistant c-FLIP, respectively. Compared with wild-type cells, c-FLIP mRNA was significantly reduced in $\Delta \mathrm{c}-\mathrm{FLIP}_{\mathrm{L} / \mathrm{S}}$ and $\Delta \mathrm{c}$ FLIP $_{\mathrm{L} / \mathrm{S}}+\mathrm{c}-\mathrm{FLIP}_{\mathrm{RESIST}}$ cells. Amplification of cFLIP $_{\text {RESIST }}$ mRNA was only detectable in cells transduced with the re-expression construct (Fig. 4a), showing that the inserted mutations were sufficient to prevent mRNA degradation by $c$-FLIP $\mathrm{L}_{\mathrm{S}}$-targeting shRNA. On the protein level, expression of $\mathrm{c}^{-F_{L}} \mathrm{P}_{\mathrm{S}}$ was downregulated in $\Delta \mathrm{c}$-FLIP $\mathrm{F}_{\mathrm{L} / \mathrm{S}}$ and $\Delta \mathrm{c}$-FLIP L $/ \mathrm{S}+\mathrm{c}$-FLIP RESIST cells, but levels of c-FLIP $\mathrm{L}_{\mathrm{L}}$ were considerably higher in $\Delta \mathrm{c}$ FLIP $_{\mathrm{L} / \mathrm{S}}+\mathrm{c}$-FLIP RESIST $_{\text {cells }}$ compared with wild-type cells, confirming the re-expression of c-FLIP RESIST while preventing expression of endogenous c-FLIP (Fig. 4b). To proof functionality of the re-expressed c-FLIP RESIST $_{\text {, we }}$ compared caspase activation and cell death rates of $\Delta \mathrm{c}$ FLIP $_{\mathrm{L} / \mathrm{S}}$ and $\Delta \mathrm{c}$-FLIP $\mathrm{L} / \mathrm{S}+\mathrm{c}$-FLIP $\mathrm{RESIST}$ cells. Caspase-8 was not activated in $\Delta \mathrm{c}-$ FLIP $_{\mathrm{L} / \mathrm{S}}+\mathrm{c}$-FLIP $\mathrm{RESIST}$ cells (Fig. 4b). In addition, intracellular active caspase-3 was 


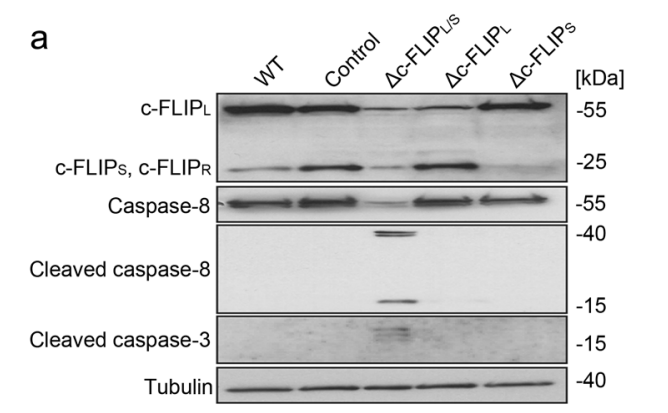

b
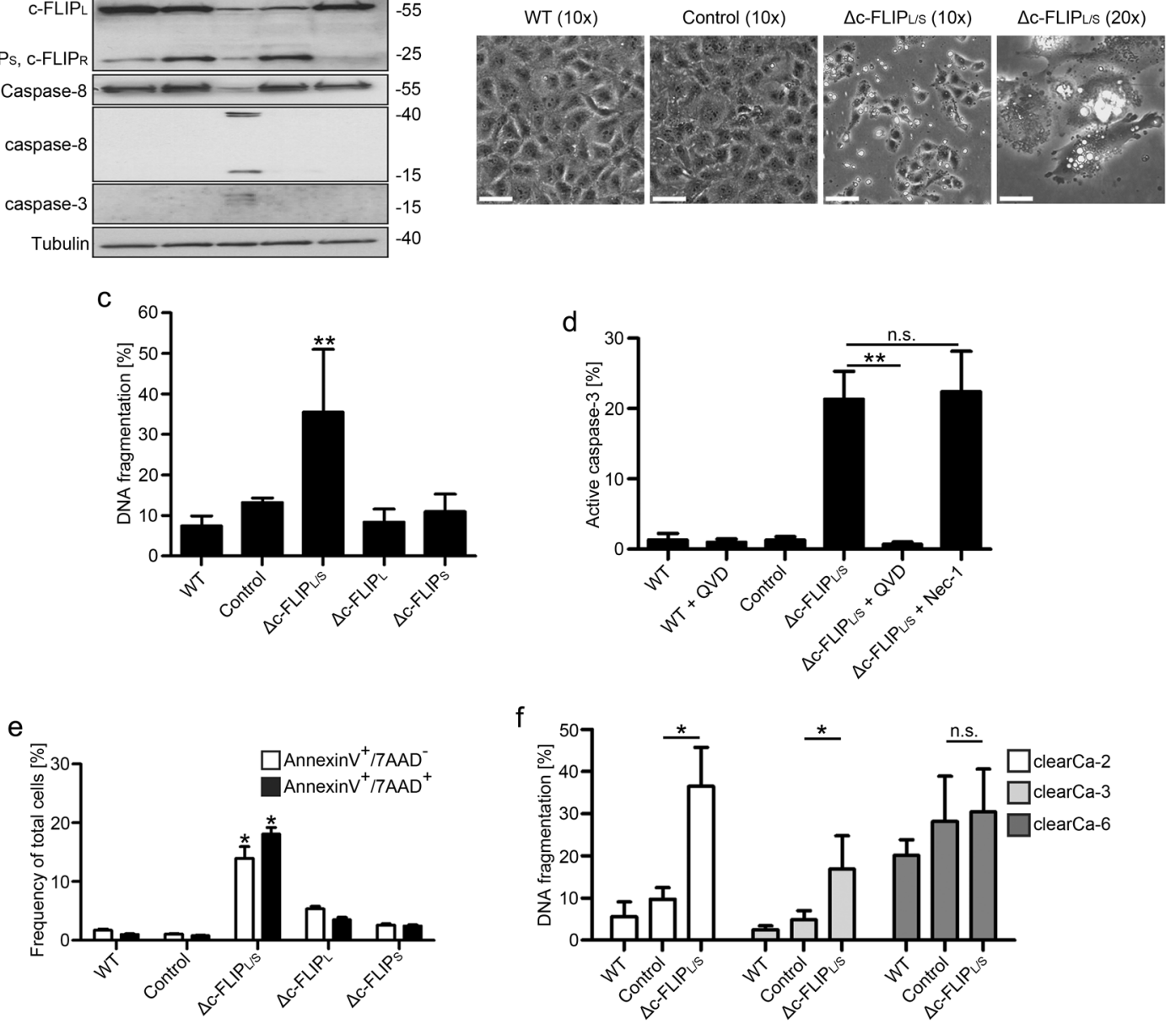

Fig. 3 Knockdown of c-FLIP induces spontaneous apoptosis in clearCa cells. a clearCa-4 cells were transduced with lentiviral constructs

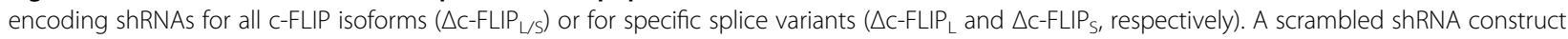
was used as control. Efficiency of c-FLIP knockdown and caspase activation was verified by immunoblot analysis three days after lentiviral transduction. b Microscopic pictures were taken on day 3 after lentiviral transduction of scrambled shRNA (control) or shRNA for all c-FLIP isoforms $(\triangle c$-FLIPL/S). White bar represents $100 \mu \mathrm{m}$ ( $\times 10$ magnification) or $50 \mu \mathrm{m}$ ( $\times 20$ magnification). c clearCa-4 cells were transduced as in a. Cell death was assessed on day 3 post transduction by analysis of DNA fragmentation. $\mathbf{d}$ clearCa-4 cells were transduced with a lentiviral construct encoding shRNA for knockdown of all c-FLIP isoforms ( $\triangle \mathrm{C}$-FLIPL/S). QVD and Nec-1 was added to block caspase-mediated apoptosis and Ripk1-mediated necroptosis, respectively. Subsequently, cells stained for intracellular active caspase-3. e clearCa-4 cells were transduced as in a and cell death was analyzed by AnnexinV/7AAD staining. f Analysis of cell death in clearCa-2, -3, and -6 cells after lentiviral knockdown of all c-FLIP isoforms on day 3 after transduction. A scrambled shRNA construct was used as control. Cell death was analyzed by quantifying DNA fragmentation via flow cytometry. Bars display the mean of at least three experiments, error bars represent SD. Statistical significances were calculated by one-tailed Mann-Whitney $U$ test in respect to Control sample; ${ }^{*} p \leq 0.05$

significantly reduced upon re-expression of c-FLIP ${ }_{\text {RESIST }}$ in comparison with $\Delta \mathrm{c}-\mathrm{FLIP}_{\mathrm{L} / \mathrm{S}}$ cells (Fig. 4c). Vitality of the cells was measured via DNA fragmentation, which revealed that re-expression of c-FLIP RESIST $_{\text {was sufficient }}$ to restore cell viability in $\Delta \mathrm{c}$-FLIP $\mathrm{L}_{\mathrm{L} / \mathrm{S}}+\mathrm{c}$-FLIP RESIST $_{\text {T }}$ cells to wild-type levels (Fig. 4d). Taken together, c-FLIP reexpression rescued the $\Delta \mathrm{c}-\mathrm{FLIP}_{\mathrm{L} / \mathrm{S}}$ phenotype of spontaneous cell death.
The CD95 receptor system is activating the NF-KB and ERK pathways and crucial for cell survival

Next to apoptosis regulation, CD95 and c-FLIP were also shown to activate NF- $\mathrm{KB}$ and $\mathrm{ERK}^{22-24,36-38}$ and CD95 expression is high in all clearCa cell lines (Fig. 1a). Therefore, we wanted to know whether clearCa cells utilized the CD95 pathway for induction of the NF- $\mathrm{kB}$ and MAP kinase survival pathways. First, we analyzed CD95L 

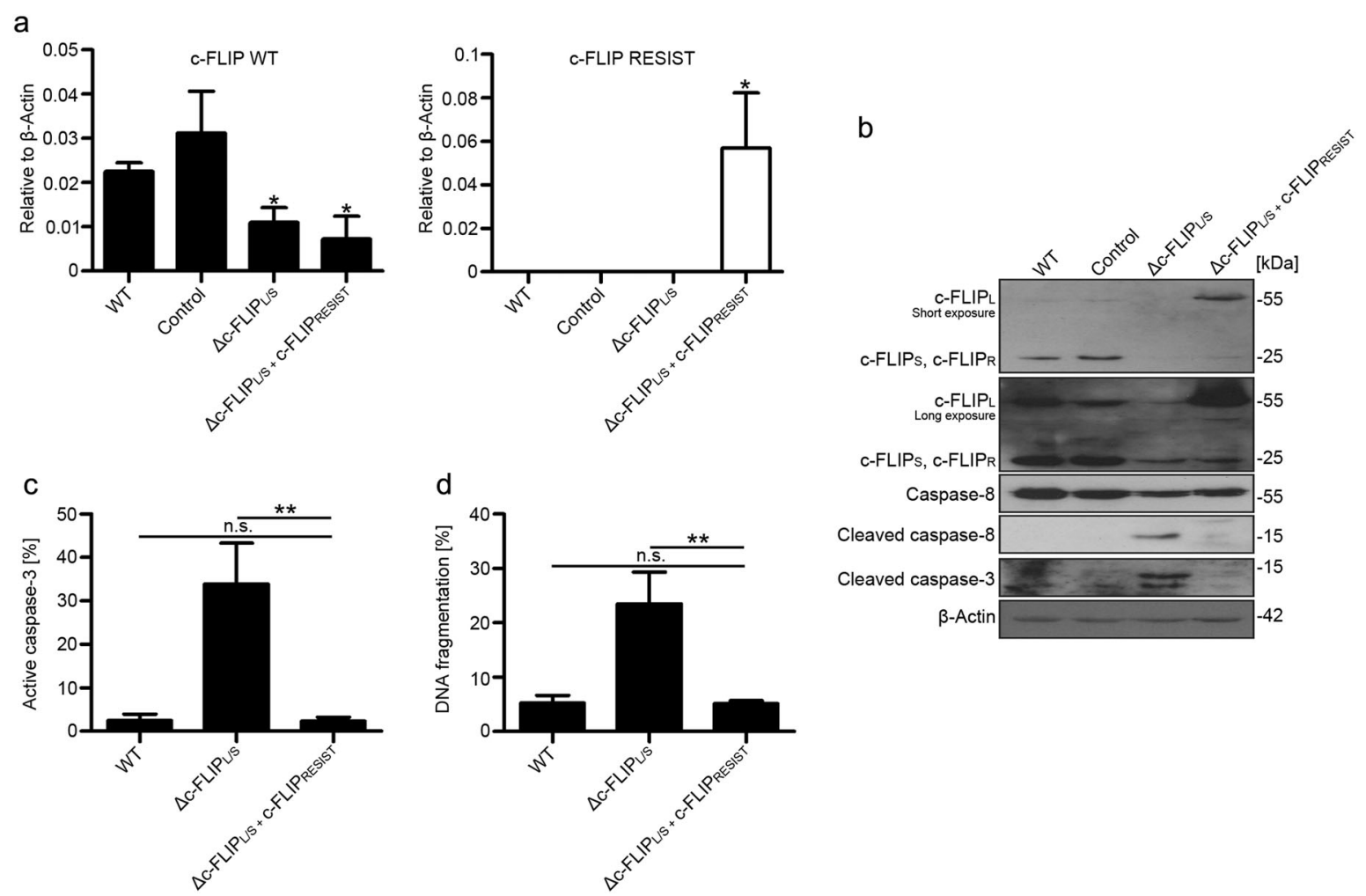

Fig. 4 Re-expression of c-FLIP $P_{L}$ protects clearCa-4 cells from c-FLIP knockdown-induced apoptosis. a Cells were transduced with either a control, a C-FLIP $/$ s/targeting, or a C-FLIPL/S targeting lentiviral construct that simultaneously expresses a shRNA-resistant version of C-FLIPL (CFLIP RESIST). Transduced cells were analyzed 3 days after lentiviral shRNA delivery. Expression of C-FLIP mRNA was analyzed with specific primers for wild type or mutated c-FLIP mRNA. Bars display the mean of three experiments, error bars represent SD; ${ }^{*} p \leq 0.05$. $\mathbf{b}$ Cells were transduced as in $\mathbf{a}$. Immunoblot analysis of c-FLIP expression levels and activation of caspase-3 and caspase-8. $\beta$-Actin was used as loading control. c Cells were transduced as in $\mathbf{a}$ and subsequently analyzed for the expression of intracellular active caspase-3 by flow cytometry. $\mathbf{b}$ Cell were transduced as in $\mathbf{a}$ and cell death was analyzed via flow cytometrical quantification of DNA fragmentation. Bars display the mean of three experiments, error bars represent SD. Statistical significances were calculated by one-tailed Mann-Whitney $U$ test; n.s. $=$ not significant, ${ }^{* *} p \leq 0.01$

surface expression by flow cytometry. ClearCa-4 cells expressed high levels of CD95L, but not TRAIL (Fig. 5a). Similarly, the expression level of CD95L was high in clearCa-2, -3, and -6 cells (Fig. 5b). In addition, CD95 surface expression pattern was analyzed via fluorescence microscopy. Interestingly, CD95 is clustered at cell-cell contacts as can be seen by the staining intensity at the contact areas of two cells (Fig. 5c). As clusters of CD95 may lead to DISC formation and subsequent cFLIP-dependent activation of survival pathways, we analyzed phosphorylation of the RelA/p65 NF-kB subunit as well as the MAP kinases Erk1/2. Phosphorylation of RelA/ p65 was already detectable in untreated cells (Fig. 5d). Stimulation with $10 \mathrm{ng} / \mathrm{mL}$ CD95L increased RelA/p65 phosphorylation, indicating NF-kB activation (Fig. 5d). Similarly, phosphorylation of the MAP kinase Erk1/2 increased upon CD95L stimulation of clearCa-4 cells (Fig. 5d).

To see if the activation of NF- $k B$ or Erk MAP kinases is necessary for cell survival, we treated ClearCa-4 cells with the IKK- $\beta$ inhibitor TPCA-1, and the MEK1 inhibitor PD0325901. Both compounds inhibited the activation of the respective target (Fig. 6a). Subsequently, cell viability was determined by resazurin assay. Importantly, TPCA-1 but not PD0325901 reduced viability of clearCa-4 cells showing that activation of IKK- $\beta$ but not Erk $1 / 2$ is required for cell survival (Fig. 6b).

c-FLIP has been shown not only to induce NF- $\mathrm{kB}$ via interaction with the IKK complex, but also to inhibit death receptor-induced NF- $\mathrm{KB}$ activity ${ }^{25-27}$. In order to investigate the function of c-FLIP in ccRCC cells, we performed knockdown experiments and analyzed the phosphorylation status of RelA/p65. Although lentiviral transduction of a scrambled shRNA into clearCa- 4 cells reduced RelA/p65 phosphorylation, $\Delta \mathrm{c}-\mathrm{FLIP}_{\mathrm{L} / \mathrm{S}}$ cells clearly had higher levels of phosphorylated RelA/p65 (Fig. 6c). Despite high NF-kB activity, $\Delta c-$ FLIP $_{\mathrm{L} / \mathrm{S}}$ cells also had high levels of cleaved caspase-8 and cleaved caspase-3. $\Delta \mathrm{c}-$ FLIP $_{\mathrm{L}}, \Delta \mathrm{c}$-FLIP $\mathrm{S}$, and $\Delta \mathrm{c}-\mathrm{FLIP}_{\mathrm{L} / \mathrm{S}}+\mathrm{c}$-FLIP $\mathrm{RESIST}$ cells exhibited an intermediate phenotype (Fig. 6c). We 

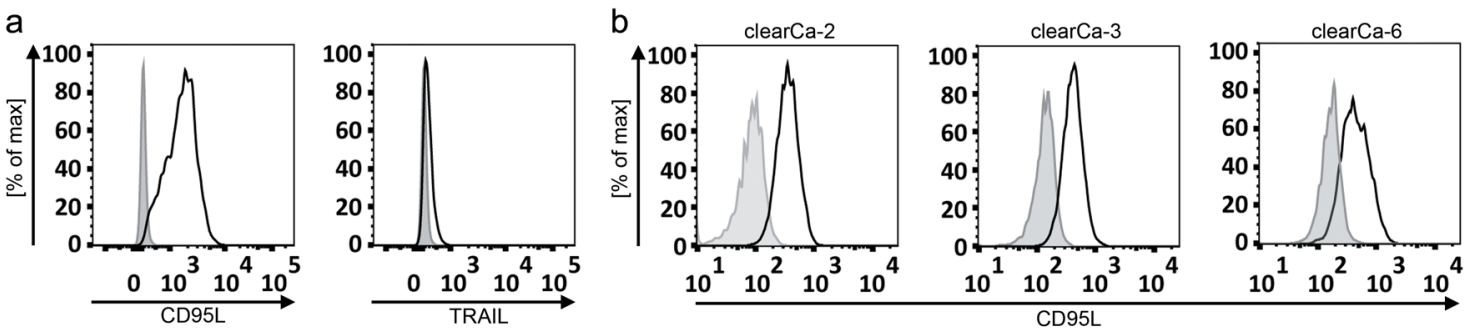

C

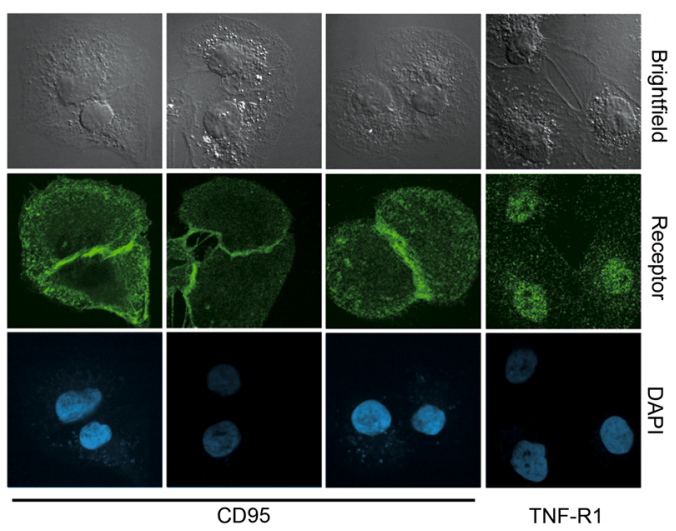

d
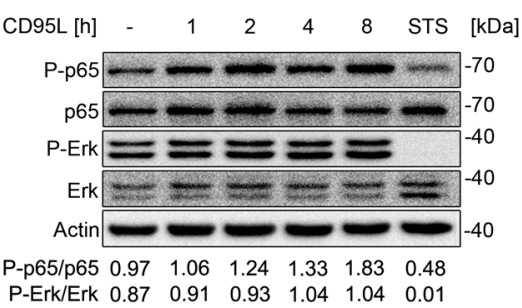

P-Erk/Erk $0.87 \quad 0.91 \quad 0.93 \quad 1.04 \quad 1.04 \quad 0.01$

Fig. 5 CD95-mediated signaling is essential for clearCa cell survival. a clearCa-4 cells were stained with antibodies against CD95L or TRAIL (black line) and analyzed by flow cytometry. Unstained samples are shown in grey. b Surface expression of CD95L (black line) was analyzed by flow cytometry on clearCa-2, clearCa-3, and clearCa-6 cells. Unstained samples are shown in grey. c CD95 and TNF-R1, respectively, were stained on clearCa-4 and analyzed by fluorescence microscopy. Receptors are depicted in green. Nuclei were stained with DAPI (blue). For each condition, 50 pictures with cell-cell contacts were analyzed; representative samples are shown. $\mathbf{d}$ clearCa-4 cells were stimulated with $10 \mathrm{ng} / \mathrm{mL} C D 95 \mathrm{~L}$ for up to $8 \mathrm{~h}$. NF-kB and MAP kinase activation was assessed by detection of phospho-p65 and phospho-Erk by immunoblot analysis. Tubulin, total p65, and total Erk were used as loading control

conclude that c-FLIP is crucial to prevent apoptosis in ccRCC cells, but additionally dampens NF-kB activity.

Finally, we wanted to know whether CD95L, CD95, and c-FLIP might be of general importance in RCC. In order to approach this question, we analyzed the expression of CFLAR (the gene encoding c-FLIP), FAS (the gene encoding CD95), and FASLG (the gene encoding CD95L) in RCC using the public data base cBioPortal $^{39,40}$. We included data sets for ccRCC ${ }^{41}$, chromophobe $\mathrm{RCC}^{42}$, and papilliary RCC (TCGA, provisional). All three genes, $C F L A R, F A S$, and FASLG, were significantly higher expressed in ccRCC compared with the other two renal cancer types (Fig. 7). Thus, targeting the CD95 pathway or c-FLIP might be a novel option for the treatment of ccRCC.

\section{Discussion}

To avoid cell death, tumors have established a broad variety of resistance mechanisms by downregulation of pro-apoptotic and upregulation of anti-apoptotic proteins including c-FLIP ${ }^{43,44}$. Accordingly, c-FLIP was shown to mediate resistance against death receptor-induced apoptosis in various tumor types ${ }^{17,28,29,34,45}$. In the current study, we focused on the role of c-FLIP-mediated CD95L resistance in $\mathrm{RCC}$.

Here, we showed that ccRCC cell lines were resistant against CD95L-induced apoptosis in the presence of cFLIP, but were sensitized upon inhibition of protein translation and subsequent reduction of c-FLIP. Accordingly, we detected activation of caspase- 8 and caspase-3, loss of cell membrane integrity and DNA fragmentation. Surprisingly, shRNA-mediated downregulation of all cFLIP splice variants was sufficient to induce spontaneous apoptosis in all ccRCC cell lines tested, without further death receptor stimulation. We confirmed that this effect was owing to the absence of c-FLIP only, as re-expression

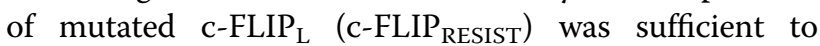
restore cell viability. Staining of death receptors revealed high levels of CD95 and CD95L on the surface of clearCa cells. Moreover, analysis of public data on the expression levels of c-FLIP (CFLAR), CD95 (FAS/TNFRSF6), and CD95L (FASLG) revealed significantly higher expression of these three genes in ccRCC compared with other renal cancers. We found that CD95, but not TNF-R1 formed clusters at cell-cell contact sites. In addition, stimulation with CD95L increased phosphorylation of RelA/p65 and 


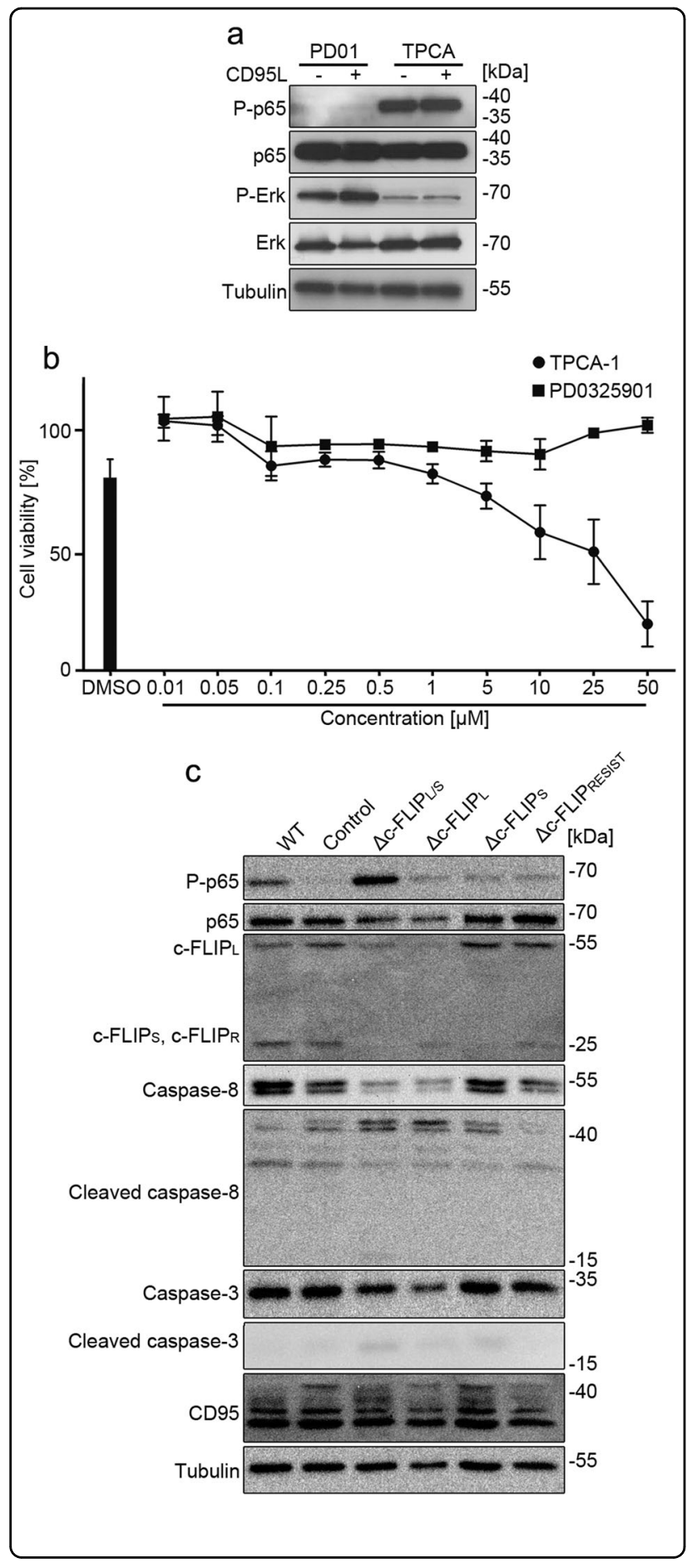

Erk1/2. Although pharmacological inhibition of Erk1/2 activation had no effect, inhibition of the NF- $\mathrm{KB}$ pathway induced cell death in clearCa cells similar to knockdown of c-FLIP and inhibition of CD95L.

The finding that knockdown of $\mathrm{c}-\mathrm{FLIP}_{\mathrm{S} / \mathrm{L}}$ alone resulted in apoptotic death of ccRCC cells was surprising as other studies reported sensitization, but not spontaneous cell
Fig. 6 CD95L-induced NF-KB is essential for clearCa cell survival and C-FLIP inhibits NF-KB in the context of CD95 signaling.

a ClearCa-4 cells were treated with $50 \mu \mathrm{m}$ TPCA-1 or $10 \mu \mathrm{m}$ PD0325901 in presence or absence of $10 \mathrm{ng} / \mathrm{ml} \mathrm{CD95L} \mathrm{for} 8 \mathrm{~h}$ and analyzed with phospho-p65 and p-Erk antibodies. Total p65, total Erk and tubulin were used as loading controls. $\mathbf{b}$ ClearCa- 4 cells were treated with the indicated concentrations of the NF-KB inhibitor TPCA-1 and the MEK1 inhibitor PD0325901, respectively, for $24 \mathrm{~h}$. Subsequently, cell viability was determined by resazurin assay. c clearCa-4 cells were transduced with lentiviral constructs encoding shRNAs for all c-FLIP isoforms ( $\Delta c$ FLIP $\left.P_{L / S}\right)$ or for specific splice variants $\left(\triangle c-F L I P_{L}\right.$ and $\Delta c-F L I P_{S}$ respectively) or a c-FLIPL/S targeting lentiviral construct that simultaneously expresses a shRNA-resistant version of C-FLIPL (cFLIPRESIST). A scrambled shRNA construct was used as control. On day 3 post transduction, cells were lysed and analyzed by immunoblotting for the indicated proteins. Tubulin was used as a loading control

death, by inhibition of c-FLIP expression. For instance, knockdown of c-FLIP resulted in higher death rates upon stimulation with death ligands in breast cancer cells ${ }^{28}$, melanoma cells ${ }^{29}$, non-small cell lung carcinoma cells ${ }^{33}$, and urothelial carcinoma cells ${ }^{34}$. To our knowledge, a single report shows that loss of c-FLIP leads to TRAILR2-dependent spontaneous caspase-8 activation and subsequent apoptosis in MCF-7 cells ${ }^{46}$, which lack caspase- $3^{47}$, a main effector caspase in apoptosis. However, the findings by Day et al. in the MCF-7 cell line are clearly different from our observations, as they claim a ligand-independent activation of caspases upon c-FLIP knockdown. In contrast, we show that ccRCC cells express high amounts of CD95L, leading to CD95 clusters at cell-cell contact sites. Furthermore, knockdown of cFLIP $_{L}$ but not c-FLIP was required for spontaneous cell death of MCF-7 cells ${ }^{46}$. For apoptosis induction in clearCa cells, complete loss of c-FLIP was necessary. Thus, c-FLIP has a special role for survival of ccRCC cells.

In this regard, we found that CD95L stimulation of clearCa cells resulted in the activation of the NF- $\mathrm{kB}$ and MAP kinase survival pathways. Pharmacological analyses demonstrated that clearCa cells depend on NF- $\mathrm{kB}$ signaling. Our observations are consistent with a recent report that showed inhibition of proliferation and growth in soft agar of ccRCC cells upon treatment with a different IKK inhibitor than the one used in our study ${ }^{48}$. Interestingly, c-FLIP has been shown to activate NF- $\mathrm{KB}^{22-24,36}$, but it is also a NF- $\mathrm{KB}$ target gene itself ${ }^{49}$. This could suggest that activation of NF-KB via the CD95L-CD95-cFLIP axis might engage a positive feedback loop to ensure protection from death receptor-mediated apoptosis. However, knockdown of c-FLIP led to an increase in RelA/p65 phosphorylation, indicating that c-FLIP inhibits NF- $\mathrm{BB}$ in ccRCC, which is consistent with reports showing an inhibitory role of c-FLIP on $\mathrm{NF}-\mathrm{kB}$ in the context of death receptor signaling ${ }^{25-27}$. Therefore, the CD95L-CD95 system appears to activate 
a

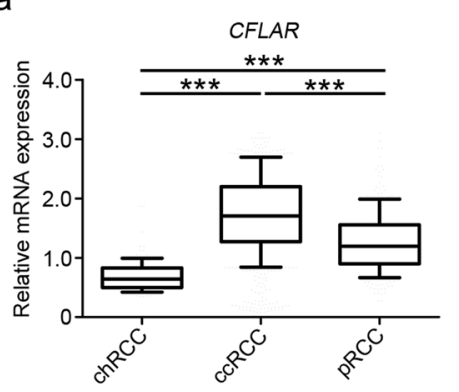

b

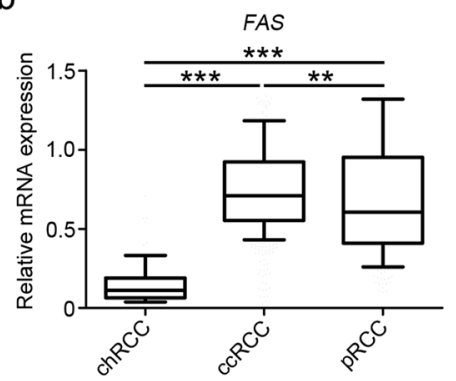

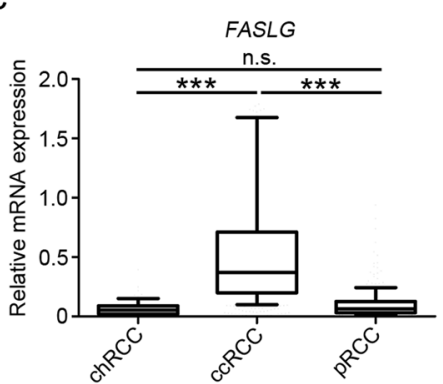

Fig. 7 CFLAR, FAS, and FASLG are differently expressed in renal cell carcinomas. a Relative expression of CFLAR determined by RNASeq V2 of clear cell renal cell carcinoma (ccRCC, $n=1003)$, kidney chromophobe (chRCC, $n=132$ ) and kidney renal papillary cell carcinoma (pRCC, $n=291)$. b Relative expression of FAS determined by RNASeq V2 of clear cell renal cell carcinoma (ccRCC, $n=534$ ), kidney chromophobe (chRCC, $n=66$ ) and kidney renal papillary cell carcinoma (pRCC, $n=291$ ). c Relative expression of FASLG determined by RNASeq V2 of clear cell renal cell carcinoma (ccRCC, $n=534$ ), kidney chromophobe (chRCC, $n=66$ ) and kidney renal papillary cell carcinoma (pRCC, $n=291)$. Box plots with whiskers (10-90 percentiles) display expression of the respective genes in chRCC, ccRCC and pRCC. Kruskal-Wallis one-way ANOVA tests were used for statistical analysis; ${ }^{* *} p<0.01,{ }^{* * *} p<0.001$, n.s. $=$ not significant

NF-kB-dependent survival signaling in ccRCC cells, whereas c-FLIP blocks CD95L-induced apoptosis and dampens NF- $\kappa B$ activity. Nevertheless, $c-F L I P$ allows NF$\mathrm{kB}$ activation up to a level that is sufficient for expression of survival factors. Next, to c-FLIP-blocking apoptosis, additional NF- $\mathrm{kB}$ target genes might affect proliferation, angiogenesis, and other processes that support tumor progression $^{50,51}$.

Although CD95 was originally regarded solely as a death receptor $^{52}$, recent evidence suggested that it can have other, non-apoptotic functions ${ }^{53}$. For instance, it was shown that CD95 can actually promote tumor formation in mouse models of liver cancer and ovarian cancer and knockdown of CD95 in cancer cell lines impaired their proliferation $^{54}$. In this regard, elevated CD95 expression was linked to later stages of RCC tumor progression, suggesting a beneficial effect of death receptor-mediated signaling for tumor cells ${ }^{55-58}$. Interestingly, we were not able to generate clearCa cells with a stable knockdown of CD95 (data not shown). At first, this latter observation is reminiscent of the recently described death induced by CD95 or CD95 ligand elimination (DICE) ${ }^{59}$. Indeed, there are certain similarities between DICE and the cell death we observe upon c-FLIP knockdown in ccRCC cells. For instance, DICE is associated with DNA fragmentation, vacuolization of the cells and caspase activation ${ }^{59}$. However, there are also clear differences. As such, DICE progresses independent of caspase activation, as it still occurs when caspases are blocked or in caspase-8deficient cells ${ }^{59}$. Moreover, DICE was described as a necrotic form of cell death. In contrast, the cell death we observed upon c-FLIP knockdown in clearCa cells exhibited classical signs of apoptosis such as caspase activation, phosphatidylserine exposure, and formation of apoptotic bodies. Furthermore, c-FLIP knockdowninduced cell death of ccRCC cells was inhibited by the pan-caspase inhibitor QVD but not by the necroptosis inhibitor Nec-1. Our data are rather in line with a study on lung adenocarcinomas, which showed that targeting CD95, c-FLIP, or NF- $\mathrm{BB}$ signaling by RNA interference sensitized lung cancer cells towards EGFR tyrosine kinase inhibitors ${ }^{60}$. Thus, also other cancer cell types might use a CD95-c-FLIP-NF- $\mathrm{kB}$ circuit for promotion of survival and growth. Nevertheless, ccRCC cells appear to be special as depletion of c-FLIP or inhibition of CD95 signaling alone was sufficient to induce cell death in these cells.

Summarized, we demonstrated that c-FLIP mediates resistance to $\mathrm{CD} 95 \mathrm{~L}$-induced apoptosis and that loss of cFLIP leads to spontaneous apoptosis in RCC. In addition, loss of CD95 signaling also drives cells into spontaneous apoptotic cell death. We suggest a model in which paracrine CD95 stimulation leads to NF- $\mathrm{BB}$ activation to promote tumor growth in clearCa cells. The function of $\mathrm{c}$ FLIP is to prevent CD95L-induced apoptosis. Although cFLIP dampens NF- $\mathrm{KB}$ activation, it still allows a level of NF- $\mathrm{kB}$ activity that is sufficient to allow for expression of survival genes. Upon loss of this pathway, cells undergo spontaneous apoptosis owing to missing pro-survival signals. Our findings that c-FLIP and CD95 are important key regulators in survival of ccRCC cells might be used for new therapeutic approaches to overcome apoptosis resistance in $\mathrm{RCC}$ by downregulating their expression. This approach is independent of cell deathactivating agents, minimizing side effects on normal tissue and might be also helpful for treatment for other tumor types. 


\section{Materials and methods Reagents}

Recombinant Flag-tagged CD95L was produced by transient transfection of HEK293T cells. The concentration of CD95L in the supernatant was determined by quantitative immunoblotting using purified Flag-FasL as a standard. CHX was purchased from Sigma Aldrich, QVD-oph was from MP Biomedicals and Nec-1 was from Enzo Life Sciences. The MEK1 inhibitor PD0325901 and the IKK2 inhibitor TPCA were purchased from Biozol/TargetMol.

\section{Cell culture and transient transfections}

Immortalized ccRCC cell lines were generated from RCC tumor dissections and have been described previously $^{35,61,62}$. RCC cell lines clearCa-2, 3, 4, and 6, and human embryonic kidney cells (HEK293T) were cultured in Dulbecco's modified Eagle's medium (DMEM high glucose; Invitrogen), supplemented with $10 \%$ fetal calf serum (PAA Laboratories), $50 \mathrm{U} / \mathrm{mL}$ penicillin, and $50 \mu \mathrm{g} /$ $\mathrm{mL}$ streptomycin. Transient transfection of HEK $293 \mathrm{~T}$ cells was done with $\mathrm{HBS}(0.28 \mathrm{M} \mathrm{NaCl}, 0.05 \mathrm{M}$ HEPES, $1.5 \mathrm{mM} \mathrm{Na}_{2} \mathrm{HPO}_{4}, \mathrm{pH} 7.0$ ) and $2.5 \mathrm{M} \mathrm{CaCl}_{2}$ according to standard protocols. Lentivirus-containing supernatant was harvested after 48 and $72 \mathrm{~h}$ post transfection.

\section{Lentiviral infection of cells}

c-FLIP MISSION TRC shRNA constructs specific for all isoforms $\left(\Delta c-F_{L} P_{L / S}\right)$ or specific for c-FLIP $\left(\Delta c-F_{L} P_{L}\right)$ were purchased from Sigma. The generation of the cFLIP $_{S}$-specific shRNA construct $\left(\Delta c\right.$-FLIP $\left.{ }_{S}\right)$ and the procedure of lentiviral transduction was previously described $^{34,63}$. In brief, lentiviral vectors were co-transfected with the envelope vector pMD2.G (Addgene no. 12259) and the gag-pol expression plasmid pCMV_dR8.2dvpr (Addgene no. 8455) into HEK293T cells as described above. The supernatant was filtered through $0.45 \mu \mathrm{m}$ polyvinylidene fluoride (PVDF) filters (GE Healthcare) and stored at $4{ }^{\circ} \mathrm{C}$. For c-FLIP isoform-specific knockdown clearCa cells were transduced with $100 \mu \mathrm{L}$ lentivirus-containing supernatant and $5 \mu \mathrm{g} / \mathrm{mL}$ polybrene (Sigma Aldrich). Cells were analyzed 3 days post transduction if not stated otherwise.

\section{Generation of a c-FLIP RESIST re-expression plasmid}

The puromycin resistance cassette of pLKO.1, containing c-FLIP $_{\mathrm{L} / \mathrm{S}}$-specific shRNA (Sigma Aldrich) was exchanged by a mutated c-FLIP gene sequence (c-FLI$\mathrm{P}_{\text {RESIST }}$, avoiding the targeting by the $\mathrm{c}-\mathrm{FLIP}_{\mathrm{L} / \mathrm{S}}$-specific shRNA to re-express functional c-FLIP ${ }_{\mathrm{L}}$. The mutated cFLIP $_{\mathrm{L}}$ gene was generated with the following primers: cFLIPL_fwd: 5'-CGAGGATCCACCGGAGCTTACCATG TCTGCTGAAGTCATCC-3'; c-FLIPL_fwd2: 5' - CGAGG ATCCACCGGAGCTTAC-3'; c-FLIPL_rev: 5' - GCTGGT
ACCTTATGTGTAGGAGAGGATAAG-3'; c-FLIP_mut _fwd: 5' - CCCTCACTTGGTCAGCGACTATAG-3'; cFLIP_mut_rev: 5' - CTATAGTCGCTGACCAAGTGAGG G-3'. Gene mutation was performed by PCR with Phusion Flash II DNA polymerase (ThermoFisher Scientific). For cloning, the amplified c-FLIP RESIST $_{\text {gene was cleaved with }}$ the restriction enzymes KpnI and BamHI (New England Biolabs).

\section{Immunoblotting}

Cells were harvested with Trypsin-EDTA (ethylenediaminetetraacetic acid; Gibco ${ }^{\circledast}$ - Life technologies,) and lysed with TPNE buffer ( $1 \% \mathrm{v} / \mathrm{v}$ Triton X-100 and $1 \mathrm{~mm}$ EDTA in phosphate-buffered saline (PBS) adjusted to $300 \mathrm{~mm} \mathrm{NaCl}$ ), supplemented with $1 \mathrm{~mm}$ phenylmethylsulfonyl fluoride, $1 \mu \mathrm{g} / \mathrm{mL}$ leupeptin, aprotinin, chymostatin, and pepstatin A. Protein concentrations were determined by Pierce BCA Protein Assay Kit (ThermoFisher Scientific) and up to $40 \mu \mathrm{g}$ protein content were loaded on a $12 \%$ polyacrylamide gel and proteins separated by their molecular weight. After transfer onto a PVDF membrane (GE Healthcare), the membrane was blocked with $5 \%$ non-fat dry milk in TBS supplemented with $0.05 \%$ Tween-20 (TBS-T) and incubated with primary antibodies overnight at $4{ }^{\circ} \mathrm{C}$. After washing with TBS-T, the membrane was incubated with secondary antibodies, coupled with horseradish peroxidase for one hour at room temperature. After a second washing step, the membrane was developed by chemiluminescence (GE Healthcare). For reusing, the blots were stripped with ReBlot Plus solution (Millipore). Primary antibodies for specific detection of proteins were: $\beta$-Actin (Ac-74, Sigma Aldrich), Bcl-x (Polyclonal, Transduction Laboratories), Caspase-8 (12F5, Dr. Klaus Schulze-Osthoff, Tübingen), Caspase-3 (Polyclonal, R\&D Systems), CD95 (C-20, Santa Cruz), c-FLIP (NF6, Adipogen), Cleaved Caspase-3 (Asp175, 9661, Cell Signaling Technology), Cleaved Caspase-8 (18C8, Cell Signaling Technology), FADD (1F7, Upstate), FADD (TA332936, Origene), PARP (4C10-5, BD Biosciences), phospho-Erk1/2 (4370, Cell Signaling Technology), phospho-p65 (3033, Cell Signaling Technology), Tubulin (DM-1A, Sigma Aldrich), XIAP (48, BD Bioscience). Horseradish peroxidase-coupled secondary antibodies: anti-mouse IgG (sc-2055, Santa Cruz), antimouse IgG1 (1070-05, SouthernBiotech), anti-mouse IgG2a (1080-05, SouthernBiotech), anti-mouse IgG2b (1090-05, SouthernBiotech), anti-goat IgG (6160-05, SouthernBiotech), anti-rabbit IgG (4030-05, SouthernBiotech), anti-rat IgG (3050-05, SouthernBiotech).

\section{Quantitative real-time PCR}

For detection of c-FLIP mRNA, cells were harvested and RNA isolated by RNeasy Plus mini Kit (Qiagen). After generation of cDNA with RevertAid RT Kit 
(ThermoFisher Scientific), real-time PCR was carried out with the $2 \times$ SYBR Green Kit (Roche) with c-FLIP-specific primers in a LightCycler 96 System (Roche). $\beta$-Actin was used as reference gene. Primers: c-FLIP_WT_fwd: 5'AACCCTCACCTTGTTTCG-3'; c-FLIP_MUT_fwd: 5'AACCCTCACTTGGTCAGC-3'; c-FLIP_rev: 5'-AACT CAACCACAAGGTCCA-3'; $\beta$-Actin_fwd: 5'-TGTTAC CAACTGGGACGACA-3'; $\beta$-Actin_rev 5'-TCTCAGCT GTGGTGGTGAAG-3'.

\section{Flow cytometry}

Cell surface staining was described previously ${ }^{34}$. For detection of dead cells, the DNA of untreated, CD95Ltreated or lentiviral-transduced cells were stained with propidium iodide and measured in the PE channel. To assess apoptotic cells, the PE Active Caspase-3 Apoptosis Kit (BD Biosciences) was used, or cells were stained with AnnexinV (BD Biosciences) and 7AAD (Enzo Life Science) to discriminate between apoptotic and necrotic cells. For surface staining, specific antibodies against CD95 (2R2, Dr. K. Schulze-Osthoff, Tübingen, Germany), CD95L (5G51, Dr. K. Schulze-Osthoff, Tübingen, Germany), TNF-R1 (H398, Dr. H. Wajant, Würzburg, Germany), TRAIL (2E5, Enzo), TRAIL-R1 (DJR1, Biolegend) and TRAIL-R2 (DJR2-4, Biolegend) were used and detected with PE-coupled anti-mouse IgG-PE (115-116-146, Jackson Immuno Research Inc.).

\section{Fluorescence microscopy}

Cells were seeded on microscopy glass coverslips and fixed with $3 \%$ paraformaldehyde. After washing with PBS, the coverslips were incubated in primary antibody solution overnight at $4{ }^{\circ} \mathrm{C}$. As primary antibodies anti-CD95 (2R2, Dr. K. Schulze-Osthoff, Tübingen, Germany) and anti-TNF-R1 (H398, Dr. H. Wajant, Würzburg, Germany) were used. After washing the coverslips with PBS, the coverslips were incubated in secondary antibody solution (anti-mouse IgG, A-11005, ThermoFisher Scientific) for1 hour at room temperature. The nuclei were stained with DAPI and pictures taken with an Eclipse Ti (Nikon instruments), supplied with an UltraViewVox Spinning Disc from PerkinElmer and analyzed with Volocity 3D Image (PerkinElmer).

\section{Resazurin assay}

After washing the cells with PBS, a 10\% solution of $0.2 \mathrm{mg} / \mathrm{ml}$ Resazurin sodium salt (Sigma) in PBS was added to fresh medium. After $3 \mathrm{~h}$ incubation in the dark at $37^{\circ} \mathrm{C}$, the fluorescence was measured at a Tecan infinite 200 reader (Ex/Em 540/590 nm).

\section{Statistics}

Statistical analyses were performed with the software GraphPad Prism (GraphPad Software). Significances were calculated with one- or two-tailed nonparametric Mann-Whitney $U$ test and Kruskal-Wallis one-way analysis of variance test.

\section{Acknowledgements}

We thank Christian Kozowsky for expert technical assistance. We are grateful to Drs. Klaus Schulze-Osthoff (Tübingen) and Harald Wajant (Würzburg) for supplying antibodies and to Drs. Csaba Mahotka and Sebastian Heikaus (Düsseldorf) for providing clearCa cell lines. This work was supported by the Deutsche Krebshilfe (108962). T.L., L.S. and F.E.S. received support from the President's Initiative and Networking Fund of the Helmholtz Association of German Research Centers under contract number VH-GS-202.

\section{Conflict of interest}

The authors declare that they have no conflict of interest.

\section{Publisher's note}

Springer Nature remains neutral with regard to jurisdictional claims in published maps and institutional affiliations.

Received: 11 October 2018 Accepted: 24 April 2019

Published online: 16 May 2019

\section{References}

1. Koul, H. et al. Molecular aspects of renal cell carcinoma: a review. Am. J. Cancer Res. 1, 240-254 (2011)

2. Frew, I. J. \& Moch, H. A clearer view of the molecular complexity of clear cell renal cell carcinoma. Annu. Rev. Pathol. 10, 263-289 (2015).

3. Gerlinger, M. et al. Genomic architecture and evolution of clear cell renal cell carcinomas defined by multiregion sequencing. Nat. Genet. 46, 225-233 (2014).

4. Turajlic, S., Larkin, J. \& Swanton, C. SnapShot: renal cell carcinoma. Cell 163, 1556-1556 (2015).

5. Ljungberg, B. et al. EAU guidelines on renal cell carcinoma: 2014 update. Eur. Urol. 67, 913-924 (2015).

6. Shinohara, N. \& Abe, T. Prognostic factors and risk classifications for patients with metastatic renal cell carcinoma. Int. J. Urol. 22, 888-897 (2015).

7. Igney, F. H. \& Krammer, P. H. Death and anti-death: tumour resistance to apoptosis. Nat. Rev. Cancer 2, 277-288 (2002).

8. Schmitz, I., Kirchhoff, S. \& Krammer, P. H. Regulation of death receptormediated apoptosis pathways. Int. J. Biochem. Cell. Biol. 32, 1123-1136 (2000).

9. Kischkel, F. C. et al. Cytotoxicity-dependent APO-1 (Fas/CD95)-associated proteins form a death-inducing signaling complex (DISC) with the receptor. EMBO J. 14, 5579-5588 (1995).

10. Chang, D. W., Xing, Z., Capacio, V. L., Peter, M. E. \& Yang, X. Interdimer processing mechanism of procaspase-8 activation. EMBO J. 22, 4132-4142 (2003).

11. Donepudi, M., Mac Sweeney, A., Briand, C. \& Grutter, M. G. Insights into the regulatory mechanism for caspase-8 activation. Mol. Cell 11, 543-549 (2003).

12. Medema, J. P. et al. FLICE is activated by association with the CD95 deathinducing signaling complex (DISC). EMBO J. 16, 2794-2804 (1997).

13. Scaffidi, C., Medema, J. P., Krammer, P. H. \& Peter, M. E. FLICE is predominantly expressed as two functionally active isoforms, caspase-8/a and caspase-8/b. J. Biol. Chem. 272, 26953-26958 (1997).

14. Scaffidi, C., Schmitz, I., Krammer, P. H. \& Peter, M. E. The role of c-FLIP in modulation of CD95-induced apoptosis. J. Biol. Chem. 274, 1541-1548 (1999).

15. Djerbi, M., Darreh-Shori, T., Zhivotovsky, B. \& Grandien, A. Characterization of the human FLICE-inhibitory protein locus and comparison of the antiapoptotic activity of four different flip isoforms. Scand. J. Immunol. 54, 180-189 (2001).

16. Golks, A., Brenner, D., Fritsch, C., Krammer, P. H. \& Lavrik, I. N. c-FLIPR, a new regulator of death receptor-induced apoptosis. J. Biol. Chem. 280, 14507-14513 (2005)

17. Irmler, M. et al. Inhibition of death receptor signals by cellular FLIP. Nature $\mathbf{3 8 8}$ 190-195 (1997).

18. Ueffing, N. et al. Mutational analyses of c-FLIPR, the only murine short FLIP isoform, reveal requirements for DISC recruitment. Cell Death Differ. 15 773-782 (2008). 
19. Boatright, K. M., Deis, C., Denault, J. B., Sutherlin, D. P. \& Salvesen, G. S. Activation of caspases-8 and -10 by FLIP(L). Biochem. J. 382, 651-657 (2004).

20. Chang, D. W. et al. C-FLIP $(L)$ is a dual function regulator for caspase-8 activation and CD95-mediated apoptosis. EMBO J. 21, 3704-3714 (2002).

21. Fricker, N. et al. Model-based dissection of CD95 signaling dynamics reveals both a pro- and antiapoptotic role of c-FLIPL. J. Cell. Biol. 190, 377-389 (2010).

22. Golks, A., Brenner, D., Krammer, P. H. \& Lavrik, I. N. The c-FLIP-NH2 terminus (p22-FLIP) induces NF-kappaB activation. J. Exp. Med. 203, 1295-1305 (2006).

23. Kataoka, T. et al. The caspase-8 inhibitor FLIP promotes activation of NFkappaB and Erk signaling pathways. Curr. Biol. 10, 640-648 (2000).

24. Koenig, A. et al. The c-FLIPL cleavage product p43FLIP promotes activation of extracellular signal-regulated kinase (ERK), nuclear factor kappaB (NF-kappaB), and caspase-8 and T cell survival. J. Biol. Chem. 289, 1183-1191 (2014).

25. Iyer, A. K. et al. Antioxidant c-FLIP inhibits Fas ligand-induced NF-kappaB activation in a phosphatidylinositol 3-kinase/Akt-dependent manner. J. Immunol. 187, 3256-3266 (2011).

26. Kavuri, S. M. et al. Cellular FLICE-inhibitory protein (CFLIP) isoforms block CD95and TRAIL death receptor-induced gene induction irrespective of processing of caspase-8 or cFLIP in the death-inducing signaling complex. J. Biol. Chem. 286, 16631-16646 (2011).

27. Kreuz, S. et al. NFkappaB activation by Fas is mediated through FADD, caspase-8, and RIP and is inhibited by FLIP. J. Cell. Biol. 166, 369-380 (2004).

28. Zang, F., Wei, X., Leng, X., Yu, M. \& Sun, B. C-FLIP(L) contributes to TRAlL resistance in HER2-positive breast cancer. Biochem. Biophys. Res. Commun. 450 267-273 (2014).

29. Geserick, P. et al. Suppression of CFLIP is sufficient to sensitize human melanoma cells to TRAlL- and CD95L-mediated apoptosis. Oncogene 27, 3211-3220 (2008).

30. Dutton, A. et al. Expression of the cellular FLICE-inhibitory protein (c-FLIP) protects Hodgkin's lymphoma cells from autonomous Fas-mediated death. Proc. Natl. Acad. Sci. USA 101, 6611-6616 (2004).

31. Mathas, S. et al. c-FLIP mediates resistance of Hodgkin/Reed-Sternberg cells to death receptor-induced apoptosis. J. Exp. Med. 199, 1041-1052 (2004).

32. Wilson, T. R. et al. c-FLIP: a key regulator of colorectal cancer cell death. Cancer Res. 67, 5754-5762 (2007)

33. McLaughlin, K. A. et al. FLIP: a targetable mediator of resistance to radiation in non-small cell lung cancer. Mol. Cancer Ther. 15: 2432-2441 (2016).

34. Ewald, F. et al. The role of c-FLIP splice variants in urothelial tumours. Cell Death Dis. 2, e245 (2011)

35. Ramp, U. et al. Apoptosis induction in renal cell carcinoma by TRAlL and gamma-radiation is impaired by deficient caspase-9 cleavage. Br. J. Cancer $\mathbf{8 8}$ 1800-1807 (2003).

36. Baratchian, M. et al. Distinct activation mechanisms of NF-kappaB regulator inhibitor of NF-kappaB kinase (IKK) by isoforms of the cell death regulator cellular FLICE-like inhibitory protein (cFLIP). J. Biol. Chem. 291, 7608-7620 (2016).

37. Legembre, P. et al. Induction of apoptosis and activation of NF-kappaB by CD95 require different signalling thresholds. EMBO Rep. 5, 1084-1089 (2004).

38. Paulsen, M. et al. Modulation of CD4+T-cell activation by CD95 co-stimulation. Cell Death Differ. 18, 619-631 (2011).

39. Cerami, E. et al. The cBio cancer genomics portal: an open platform for exploring multidimensional cancer genomics data. Cancer Discov. 2, 401-404 (2012).

40. Gao, J. et al. Integrative analysis of complex cancer genomics and clinical profiles using the cBioPortal. Sci. Signal. 6, pl1 (2013).
41. The Cancer Genome Atlas Research Network. Comprehensive molecular characterization of clear cell renal cell carcinoma. Nature 499, 43-49 (2013).

42. Davis, C. F. et al. The somatic genomic landscape of chromophobe renal cell carcinoma. Cancer Cell. 26, 319-330 (2014).

43. Fulda, S. Targeting C-FLICE-like inhibitory protein (CFLAR) in cancer. Expert. Opin. Ther. Targets. 17, 195-201 (2013).

44. Kataoka, T. The caspase-8 modulator c-FLIP. Crit. Rev. Immunol. 25, 31-58 (2005).

45. Troeger, A. et al. Up-regulation of c-FLIPS+R upon CD40 stimulation is associated with inhibition of CD95-induced apoptosis in primary precursor B-ALL. Blood 110, 384-387 (2007).

46. Day, T. W., Huang, S. \& Safa, A. R. c-FLIP knockdown induces ligandindependent DR5-, FADD-, caspase-8-, and caspase-9-dependent apoptosis in breast cancer cells. Biochem. Pharmacol. 76, 1694-1704 (2008).

47. Janicke, R. U., Sprengart, M. L., Wati, M. R. \& Porter, A. G. Caspase-3 is required for DNA fragmentation and morphological changes associated with apoptosis. J. Biol. Chem. 273, 9357-9360 (1998).

48. Zhang, J. et al. VHL substrate transcription factor ZHX2 as an oncogenic driver in clear cell renal cell carcinoma. Science 361, 290-295 (2018).

49. Micheau, O., Lens, S., Gaide, O., Alevizopoulos, K. \& Tschopp, J. NF-kappaB signals induce the expression of c-FLIP. Mol. Cell. Biol. 21, 5299-5305 (2001).

50. Basseres, D. S. \& Baldwin, A. S. Nuclear factor-kappaB and inhibitor of kappaB kinase pathways in oncogenic initiation and progression. Oncogene $\mathbf{2 5}$ 6817-6830 (2006).

51. Perkins, N. D. The diverse and complex roles of NF-kappaB subunits in cancer. Nat. Rev. Cancer 12, 121-132 (2012).

52. Krammer, P. H. CD95's deadly mission in the immune system. Nature 407, 789-795 (2000).

53. Peter, M. E. et al. The CD95 receptor: apoptosis revisited. Cell 129, 447-450 (2007).

54. Chen, L. et al. CD95 promotes tumour growth. Nature 465, 492-496 5 (2010).

55. Horie, S. et al. Expression of Fas in renal cell carcinoma. Jpn. J. Clin. Oncol. 27 384-388 (1997).

56. Macher-Goeppinger, S. et al. Expression and prognostic relevance of the death receptor CD95 (Fas/APO1) in renal cell carcinomas. Cancer Lett. 301, 203-211 (2011).

57. Sejima, T. \& Miyagawa, I. Significance of Fas expression alteration during tumor progression of renal cell carcinoma. Int. J. Urol. 13, 257-264 (2006).

58. Sejima, $T$. et al. Fas expression in renal cell carcinoma accurately predicts patient survival after radical nephrectomy. Urol. Int. 88, 263-270 (2012).

59. Hadji, A. et al. Death induced by CD95 or CD95 ligand elimination. Cell Rep. 7, 208-222 (2014)

60. Bivona, T. G. et al. FAS and NF-kappaB signalling modulate dependence of lung cancers on mutant EGFR. Nature 471, 523-526 (2011).

61. Heikaus, S., Kempf, T., Mahotka, C., Gabbert, H. E. \& Ramp, U. Caspase-8 and its inhibitors in RCCs in vivo: the prominent role of ARC. Apoptosis 13, 938-949 (2008).

62. Ramp, U. et al. Deficient activation of CD95 (APO-1/Fas)-mediated apoptosis: a potential factor of multidrug resistance in human renal cell carcinoma. Br. J. Cancer 82, 1851-1859 (2000)

63. Ueffing, N., Schuster, M., Keil, E., Schulze-Osthoff, K. \& Schmitz, I. Up-regulation of c-FLIP short by NFAT contributes to apoptosis resistance of short-term activated T cells. Blood 112, 690-698 (2008). 